Research Paper

\title{
Enhanced thermal transport in polymers with an infrared-selective thermal emitter for electronics cooling
}

\author{
Shinichiro Tsuda*, Makoto Shimizu, Fumitada Iguchi, and Hiroo Yugami \\ Department of Mechanical System Design, Graduate School of Engineering, Tohoku University, Sendai 980-8577, Japan
}

\begin{abstract}
Passive thermal management has attracted much attention for use in electronic devices. In this study, we propose a novel thermal management technique based on spectral matching between thermal radiation and the infrared optical window of polymers, which are widely used in device encapsulation and packaging. The unique effects of this technique on heat transfer were numerically and experimentally studied. Our numerical analysis confirms that selectively enhancing thermal radiation in the optical window of polymers improved thermal propagation deep into the polymers, reducing local temperature distributions at their surface. In addition, our experiments demonstrate that boosting thermal radiation propagation deep into polymers effectively enhanced their heat spreading and dissipation. Our new method for thermal management in polymers can improve passive thermal management in electronic devices that include polymers.
\end{abstract}

Keywords: Thermal radiation control; polymers; heat transfer enhancement; passive thermal management; electronic devices

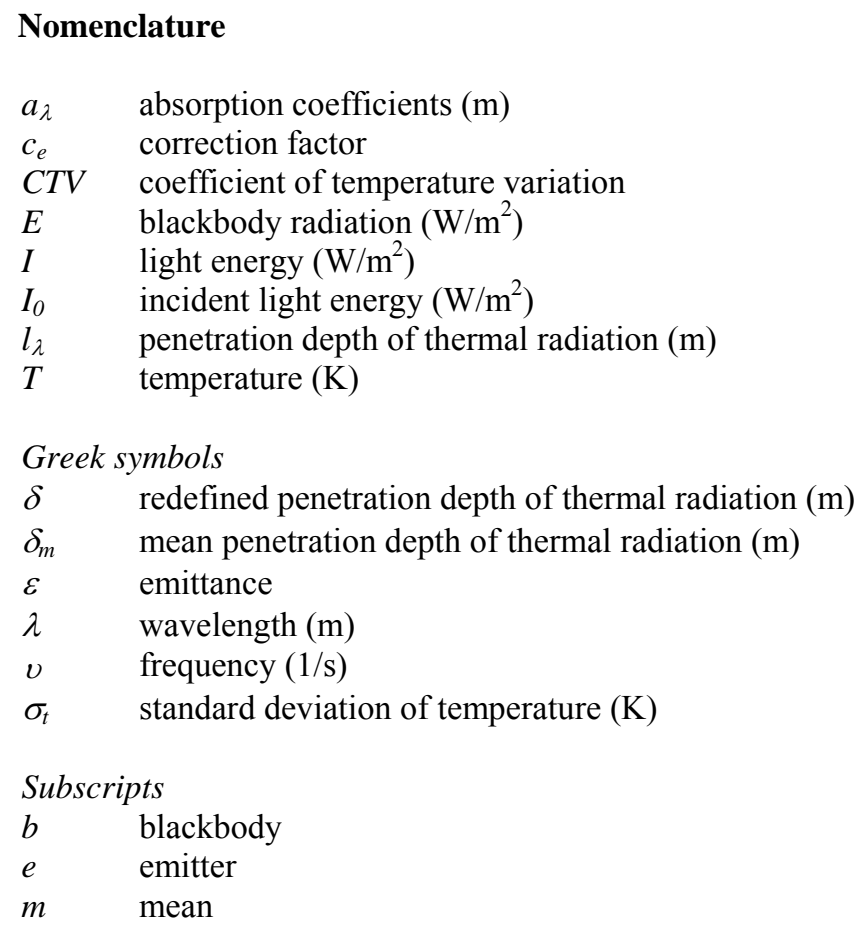

* Corresponding author. E-mail address: s_tsuda@energy.mech.tohoku.ac.jp 


\section{Introduction}

Thermal management is essential in electronic devices because of trends such as increasing the integration density of semiconductor chips, reducing cost, saving energy, and miniaturization [1]. In particular, the thermal design of electronic devices is limited by local temperature distributions, generally known as hotspots, decreasing device reliability and performance [2,3]. Although passive thermal management solutions using thermal conduction and convection are preferred in battery-powered electronic devices, these solutions sometimes require large cooling components, making them unusable in many devices. In this work, we study and develop a method of passive thermal management, not requiring a heating medium or external power for heat transport, which effectively uses thermal radiation.

Thermal radiation is the energy emitted from a hot surface as electromagnetic waves. In electronic devices, thermal radiation complements thermal conduction and convection, in turn increasing heat transfer, but radiation contributes relatively little to heat transfer at the operating temperatures of electronic devices, as shown by Planck's law. With recent progress in quantum optics and nanotechnology, spectral control of thermal radiation has drawn interest [4-14], and this technique shows promise for various applications in thermophotovoltaics [15,16], thermal imaging [17], infrared sensing [18], and radiative cooling [19,20]. In particular, research on radiative cooling with spectral control of thermal radiation, which matches the spectrum of thermal radiation and the optical window of the atmosphere $(8-13 \mu \mathrm{m})$, shows that highefficiency passive cooling systems using thermal radiation are feasible even at low temperatures $[19,20]$.

Similar to radiative cooling systems, we have also proposed a thermal management technique for electronic devices that use spectral control of thermal radiation $[21,22]$. This technique works by employing the infrared optical window of polymers, often used to encapsulate and package electronic devices. Typical polymers such as epoxy resin, acrylic resin, and polyethylene show lower absorption coefficients in the infrared wavelength region of 3.5-5.5 $\mu \mathrm{m}$. In this technique, as shown in Figure 1, heat generated from a heat source encapsulated or packaged in polymers released from the emitter, a device for spectral control of thermal radiation, as thermal radiation matched the optical window of the polymer. The spectrally selective thermal radiation propagates through the polymer into the ambient environment with low absorption loss both because of the enhanced thermal radiation in the polymer's optical window and because of the suppressed propagation at wavelengths with high absorption thanks to the spectral control. This technique is 
a passive, compact thermal management solution similar to heat pipes [23,24] and graphite sheets [25], though unlike these solutions it actively uses thermal radiation.

This technique is also unique because it can improve heat transfer in polymers, which have low thermal conductivity because of their reduced phonon mean free path (MFP), caused by their random molecular chains [26-28]. Indeed, radiative heat transfer plays an important role in heat transfer and redistribution in polymers. Thermal radiation in the semitransparent medium propagates deep into the medium, preventing increases in surface temperature, which makes it an effective heat transport mechanism compared with thermal conduction $[29,30]$. In fire science, investigations into radiative heat transfer in polymers have been conducted in hopes of understanding their ignition mechanism [29,31,32]. These studies' findings suggest that spectral control of thermal radiation by using photonics and plasmonics enhances radiative heat transfer in polymers by modulating the spectral features of the thermal radiation radiated to the polymers.

In this study, we propose a new thermal management technique for devices encapsulated in or packaged with polymers, enhancing the heat transport in the polymers by spectral control of thermal radiation. We studied how this technique enhanced heat transport and spreading in polymers both numerically and experimentally. 


\section{Numerical characterization of the proposed thermal management technique}

To evaluate how our technique affects the penetration of thermal radiation into polymers and their heat transport behavior, we propose the characteristic parameter of the mean penetration depth (MPD) of the thermal radiation. This MPD was calculated by using optical measurements to estimate the polymers' absorption coefficients. Based on this MPD, we designed and optimized a spectrally selective emitter (SSE), used for spectral control of thermal radiation. The heat transport properties of the polymers were characterized by comparing their MPDs when using an SSE and spectrally nonselective emitters.

\subsection{Description of the mean penetration depth}

The exponentially decaying intensity of thermal radiation propagating in an absorbing medium is described by Beer's law. If the medium is assumed to be spatially homogeneous, and the absorption coefficient of the medium is constant, the penetration depth ( $\mathrm{PD}$, or MFP) of the thermal radiation $l_{\lambda}$ is denoted as follows [33]:

$$
l_{\lambda}=\frac{1}{a_{\lambda}}
$$

where $l_{\lambda}$ is generally the inverse of the absorption coefficient of the medium. In this study, $l_{\lambda}$ is redefined as $\delta_{\lambda}$ following the definition by Basu et al. of the thermal radiation PD: the depth at which $95 \%$ of the thermal radiation energy is attenuated by absorption from the medium [34]. It is denoted as follows:

$$
\delta_{\lambda}=-\frac{\ln (0.05)}{a_{\lambda}}
$$

To evaluate the total transferred power, $\delta_{\lambda}$ is weighted for all wavelength regions and is extended to the MPD of thermal radiation $\delta_{m}$ to interpret the thermal radiation radiated from an emitter propagating and absorbing in the medium as follows:

$$
\frac{I\left(\delta_{m}\right)}{I_{0}}=\frac{\int_{0}^{\infty} \varepsilon_{e}(v) E_{b}\left(T_{e}, v\right) e^{-a(v) \delta_{m}} d v}{\int_{0}^{\infty} \varepsilon_{e}(v) E_{b}\left(T_{e}, v\right) d v}=0.05,
$$

This formula ignores the reflection in the interface for ease of calculation. The MPD of thermal radiation into the medium denotes an effective enhancement factor of heat transport in the medium by thermal radiation. 
Thereby, the change in the heat transport behavior in polymers caused by the penetration of thermal radiation can be evaluated with the MPD. This MPD can be improved by spectral matching between the thermal radiation spectrum and the optical window of the polymer, as will be shown later in this paper.

\subsection{Methods for evaluating the absorption coefficients of polymers}

Calculating the MPD in Eq. (3) requires the absorption coefficients of polymers over a wide range of wavelengths. However, these have not been reported well previously because originally it is complicated to make an absolute estimate of absorption coefficients that accounts for reflection in the interface, internal scattering, and concentrations. There are some ways to experimentally estimate the absorption coefficients of materials: fused quartz containing air bubbles has been evaluated using Mie theory and two-flux method [35], and thermal barrier coatings have been evaluated by fitting the measured reflectance and transmittance with the two-flux method and the Kubelka-Munk model [36]. Dubols et al. reported the absolute absorption coefficients of epoxy resin measured by photoacoustic spectroscopy [37]. However, because the data on acrylic resin assumed to be used in the present study do not appear to have not been reported well based on our best knowledge, especially over a wide range of wavelengths, the absorption coefficients of acrylic resin must be directly measured or estimated. In this study, these coefficients were estimated by combining Lambert-Beer's law and an optical measurement with the $\mathrm{KBr}$ pellet technique [38] for ease of use. Future studies should consider a more precise estimation method because our method includes uncertainties based on internal scattering by grains; however, for the purposes of this study, this estimation is likely sufficient to grasp the underlying mechanism.

\subsection{Design of the spectrally selective emitter and evaluation of its optical properties}

In this study, the SSE was used as a cooling device, which allowed us to match the thermal radiation spectrum to the optical window of the polymers. Substantial research has addressed the devices and methods used to control thermal radiation: optical phenomena in photonic crystals [4-8], in multilayer stacks $[9,10]$, surface plasmon polariton (SPP) resonance [11], surface phonon polariton in oxides [12], fluorescence of rare earth elements [13], and recently the combination of intersubband transitions in multiple quantum wells and photonic crystals [14]. In the present study, we realized spectral matching between the thermal radiation spectrum and the optical window of the polymers by using 2D metallic surface grating structures that interact with electromagnetic waves through both the microcavity effect [5] and SPP resonance, precisely tuning the 
optical absorption and emission properties. As shown in Figure 2(a), the surface grating structures consist of many microcavities, which confine only the electromagnetic waves matching the characteristic feature sizethat is, the microcavities selectively enhance emission and absorption within a particular wavelength range. In addition, because the optical windows of the polymers have certain bandwidths, the enhanced emission peak should be wide so as to enlarge the thermal radiative power in the optical window. The present structure provides better thermal radiation for thermal management applications with an optical window, because its spectral selectivity has a reasonably broad range. The effect depends on the microstructural surface features and the intrinsic optical properties of the material, so it should be optimized.

Figure 2(b) shows the optical properties of the fabricated SSE, optimized by studying the MPD of thermal radiation into the polymers, and the absorption coefficients of polymers (acrylic resin) estimated using the $\mathrm{KBr}$ pellet technique. In addition, the optical properties were measured by Fourier-transform infrared spectroscopy (FT-IR) at wavelengths of 2.5-25 $\mu \mathrm{m}$. The SSE-consisting of Si microstructures laminated with a Pt/Ti nanolayer — was fabricated by photolithography and sputtering, as described in a previous report [22]. Though it is likely that these absorption coefficients are slightly underestimated in the absorption bands of these polymers compared with those of epoxy resin [37], the values agree relatively well in their orders of magnitude: $10^{3}$ to $10^{4} \mathrm{~m}^{-1}$ in the optical window, and $10^{4}$ to $10^{5} \mathrm{~m}^{-1}$ in the absorption bands. Figure 2(b) shows that thermal radiation from the SSE was enhanced in the optical window and suppressed in the absorption bands.

\subsection{Numerical analysis of penetration depth}

To investigate how the selectively enhanced thermal radiation in the optical window of the polymers affected their heat transport, we performed a numerical analysis of the MPD of thermal radiation into the polymers, assessing the SSE and spectrally non-selective emitters, a high emissivity emitter (HEE) and a low emissivity emitter (LEE), as shown in Figures 2 and 3, respectively. Figure 4 shows a decaying thermal radiation propagating in polymers with respect to wavelength for each emitter at the emitter temperature of $100{ }^{\circ} \mathrm{C}$ derived from Eq. (3), giving the relationship between the spectral penetration depth and radiative intensity, with a simple iterative method. The radiation propagation medium is assumed to be acrylic resin, as discussed in Figure 2(b). With the SSE (Figure 4(a)), the thermal radiation in the absorption bands is not actually absorbed at the polymer surface, and in the optical window the radiation propagates deep into the 
polymer, compared with the HEE (Figure 4(b)). These results show that the thermal radiation selectively enhanced in the optical window of the polymer improved the heat transport, especially near the surface. The case with the LEE is similar to the HEE because both have spectrally non-selective radiative features. Figure 5 also shows the MPD $\delta_{m}$ directly derived from Eq. (3) for each emitter at various emitter temperatures, revealing that the SSE produced a much higher MPD than the spectrally non-selective emitter did. This figure includes the enhancement of heat transport near the polymer surface, and shows the increase in the effective propagation length of thermal radiation in the polymers by SSE. These results also show that the MPD can characterize the heat transport, including the propagation of thermal radiation, in polymers. Additionally, the MPD with emitters is enhanced by the temperature increase because the emission peak results in a blueshift with increasing temperature based on Planck's law, matching to the optical widow of the polymers. 


\section{Experimental setup to evaluate heat transport in polymers}

Here, we describe the experimental setup we used to demonstrate how applying our proposed technique improved heat transfer in polymers. In these experiments, we considered both the emissive power from various emitters and the radiative spectrum passing through polymers with various thicknesses. In addition, accounting for the impact, we characterized how the proposed technique affected heat spreading in the polymers by evaluating their surface temperature distributions.

\subsection{Experimental setup}

Figure 6 gives the experimental setup, showing the heater on the thermal insulation (Porextherm WDS, Porextherm dammstoffe GmbH, Germany) and emitters, and showing the polymer plates (acrylic resin plate, Mitsubishi Rayon, Japan), located in the vacuum chamber. In this setup, the heat transfer path is assumed to be quasi-one-dimensional, with heat conduction from the heater to the emitters, and thermal radiation from the emitters to the polymer plates, as well as through the polymer plates and the infrared optical window $\left(\mathrm{BaF}_{2}\right)$. The thermal radiation transmitted through the infrared optical window is measured by the optical system, including FT-IR, letting us characterize the thermal radiation through polymer plates with various thicknesses. Simultaneously, we evaluated the surface temperature distributions of the polymer plates by taking thermal images of them with a thermal camera (TH6200R, NEC Avio, Japan) through the infrared optical window.

To characterize the impact of thermal radiation propagating in the polymers, we used polymer plates with two thicknesses with transmission $(0.5 \mathrm{~mm})$ and less transmission $(2 \mathrm{~mm})$ at the optical window of polymers, as shown in Figure 6(c). These are made from the acrylic resin discussed in the previous section. Here we also used spectrally selective and high emissivity emitters, discussed in Figures 2 and 3. By comparing these combinations of emitters and polymer plates, we experimentally evaluated the heat transport in these polymers, studying how it was modulated by the propagation of thermal radiation.

The heater was held at a constant temperature in the range of $25-360{ }^{\circ} \mathrm{C}$, which in turn controlled the temperatures of the emitter and polymer plate. Once the temperature reached a steady state, we measured the thermal radiation spectrum through the polymer plate. To confirm the effect of heat spreading in the polymer, the temperature distributions at the polymer surface were simultaneously observed with the thermal camera. The pressure in the vacuum chamber was $\sim 1 \mathrm{~Pa}$; at this pressure, there is negligible thermal 
conduction and convection between the emitters and polymer plates, confirmed by comparing the MFP of the air in the vacuum chamber and the feature sizes of the components. In addition, because the alumina pipes supporting the polymer plates are spatially and thermally isolated from the base in Figure 6(a), the dominant heat-transfer mechanism between the emitters and polymer plates is assumed to be radiative heat transfer.

\subsection{Correction method for radiative spectra}

Before evaluating the measured radiative spectrum, we introduced a correction factor that accounts for the experimental equipment. In our experiment, we could precisely evaluate the thermal radiation spectrum only in the measured wavelength range of $2-10 \mu \mathrm{m}$. For wavelengths above $10 \mu \mathrm{m}$, there is unwanted absorption by the $\mathrm{BaF}_{2}$ or $\mathrm{CaF}_{2}$ infrared optical window. The thermal radiation spectrum over this wavelength range can be evaluated using the correction factor as follows:

$$
c_{e}=\frac{E_{e}\left(\lambda, T_{e}\right)}{E_{b}\left(\lambda, T_{e}\right)},
$$

where the numerator denotes the measured thermal radiation spectrum emitted by HEE with approximately constant emissivity in the wavelength regions, and the denominator denotes the blackbody radiation given by Planck's law at an emitter temperature. The measured thermal radiation spectrum is corrected by the division with the correction factor in Eq. (4).

\subsection{Method for evaluating hotspot reduction}

To analyze how our method affected heat spreading and hotspot reduction, we qualitatively evaluated the surface temperature distributions of the polymer plates from their thermal images, finding the standard deviations of surface temperature. This approach to analyzing heat spreading by using standard deviations is addressed in a study of a heat spreader and vapor chamber by Chen et al. [39]. Because the standard deviation of temperature of a polymer plate depends on its mean temperature, this study uses the coefficient of temperature variation (CTV), including the correction of temperatures. The CTV is described as follows:

$$
C T V=\frac{\sigma_{t}}{T_{m}}
$$


where the mean surface temperature of the polymer plate evaluated through thermal imaging is corrected by the transmittance of $\mathrm{BaF}_{2}$. The standard deviation of temperature is related to variation in surface temperature, so a lower CTV denotes better heat spreading and fewer surface hotspots.

\section{Experimental results and discussion}

\subsection{Thermal radiation spectra and enhanced heat transport}

To evaluate the impact of thermal radiation propagating in polymers, we measured radiative spectra from the emitters through the polymers, by using the optical system including FT-IR. Figure 7(a) shows the radiative spectra for each emitter and polymer plates with two thicknesses. These spectra were taken at approximately the same emissive power from each emitter surface, given by multiplying the emittance of the emitters and the blackbody radiation given by Planck's law. The emissive power is also denoted as the input power from the emitters to the polymer plates, because thermal radiation plays a dominant role in heat transfer in these components. Additionally, the absorption properties of these polymers are not likely to vary greatly with increasing temperature, as shown by comparing Figure 6(c) and Figure 7(a). Even though the absorption peaks may broaden and shift because of slight fluctuations in the rotational and vibrational transitions energy levels of polymers related to increased temperature [40], the effect of temperature on the optical window is basically negligible unless the polymers experienced thermal dissociation.

Here, the temperatures of the SSE are naturally higher than those of the HEE because the SEE has lower average emittance over the range of wavelengths considered, as shown in Figure 3. As shown in Figure 7(a), in the polymers' absorption bands, which are longer than $6 \mu \mathrm{m}$, their spectral features were similar because the thermal radiation from the emitters was totally absorbed by the polymer in this range of wavelengths, so the radiation mostly comes from the heated polymer plates. This also occurred with a polymer plate that did not allow direct optical transmission (thickness of $2 \mathrm{~mm}$ ), even within the polymer's optical window. However, by using a polymer plate that allowed direct optical transmission (thickness of $0.5 \mathrm{~mm}$ ), especially within the polymer's optical window, a difference appeared in the spectral features among the emitters because the spectra included the emissive power from the emitters transmitted through the polymer plates.

Compared with the radiative intensity of the HEE, that of the SSE within the optical window was greater because of effective spectral matching, accomplished by the spectral control of thermal radiation and the blueshift of the emission intensity peak induced by increasing the emitter temperature as mentioned in 
Section 2.4. Additionally, the radiative intensity is clearly higher for the SSE than for the HEE regardless of the plate thickness. This result suggests that thermal radiation from the SSE effectively propagated through the polymer plate with less re-emitted radiation from the polymer plate to the emitter; the increased MPD of thermal radiation into the polymers agrees with the theoretical results shown in Figures 4 and 5. Figure 7(b) shows the mean temperatures of the polymers with respect to the emissive power from the emitter. The above-mentioned radiative intensity from the heated polymer plate depends on the mean temperature of the polymer. Our experimental results show that the polymer plate is heated more efficiently by the SSE than by the HEE. These results also show the effect of effective spectral matching by the SSE.

\subsection{Improvement in heat spreading and reduction in hotspots}

Figure 8 shows thermal images at the surfaces of the polymer plates through the infrared optical window $\left(\mathrm{BaF}_{2}\right)$, considering each emitter and polymer plates of various thicknesses. For ease of comparison, the middle of the range of mean surface temperatures was set to around $100{ }^{\circ} \mathrm{C}$. The temperature profiles at the bottom of the thermal images are denoted as blue lines in the thermal images. With the SSE (Figure 8(a) and (c)), the polymer plates exhibited surface temperature distributions, comparing the center and edge, that were more uniform than those with the HEE (Figure 8(b) and (d)), at any plate thickness. These results suggest that the thermal radiation selectively enhanced in the polymer's optical window effectively enhanced heat spreading and reduced hotspots.

To quantitatively evaluate the surface temperature distributions in the polymers, these are arranged in terms of CTV from Eq. (5). Figure 9 shows the CTV with respect to the mean surface temperature of the polymer for each emitter and various plate thicknesses. The CTV with the SSE is lower than that with the HEE at all temperatures studied, showing how the SSE enhanced heat spreading and reduced hotspots. In addition, the CTV increased with the mean temperature of the polymer, caused mainly by the characteristic of radiative heat transfer between parallel plates, i.e., configuration factors [33]. Hence, the thermal radiation from the emitter comparatively concentrated on the center of the polymer plate above the emitter, and this effect increased at high temperatures. The flat regions from the thin polymer plate (thickness of $0.5 \mathrm{~mm}$ ), against the trend, likely come from direct optical transmission, which dramatically increased with increasing emitter temperature. 
Our experimental results show that the CTV decreased by a maximum $5 \%$ to $10 \%$ with the SSE compared with the HEE at all plate thicknesses, enhancing heat spreading and reducing hotspots. In comparison, for the mean surface temperature of the polymer, with the SSE the CTV hardly depended on the plate thickness; in contrast, with the HEE the CTV significantly increased as the plate thickness increased. These differences suggest that the degree of heat concentration (hotspots) varied with the emitter and plate thickness: the SSE reduced hotspots regardless of plate thickness; the HEE easily generated hotspots by increasing the absorption of thermal radiation in the polymer, especially when using polymer plates that did not directly transmit thermal radiation. Overall, boosting the MPD of thermal radiation into polymers improves their heat spreading and reduces surface hotspots, as shown in Figures 4 and 5. Additionally, the effect on heat spreading and hotspots was influenced less by the plate thickness; that is, it depended less on direct transmission of thermal radiation. These results show that enhancing the internal heat transport in polymers can enhance their heat spreading and reduce hotspots.

\section{Conclusions}

This study demonstrated a new thermal management technique based on enhancing heat transport in polymers by spectral matching between the thermal radiation and the optical window of the polymer, investigated both numerically and experimentally. To study how the spectral control of thermal radiation affected heat transport in the polymers, we used the characteristic property of MPD. To perform these calculations, we estimated the absorption coefficients of the polymers from optical measurements. The radiative cooling device, i.e., the SSE, which matches the thermal radiation to the optical window of the polymer, was optimized by MPD and fabricated by photolithography and sputtering. Our numerical analysis showed that the SSE increased the penetration depth of the thermal radiation, reducing the local temperature distributions at the polymer surface. Our experiments confirmed that our method increased heat spreading and reduced hotspots by analyzing the surface temperature distributions of polymers and the radiative spectra transmitted through the polymers. Furthermore, the radiative spectrum emitted from the emitters showed that our technique improved heat dissipation through thermal radiation. But otherwise, temperature management in actual thermal systems including emitter devices would have to be well designed, because of temperature constraints related to thermal issues. Our technique can be used for passive thermal management in electronic devices that include polymers as well as in various thermal applications that use infrared 
semitransparent materials such as glass, including in solar-cell modules [41]. Our method is a novel way to enhance heat transfer in polymers by manipulating photon transport, unlike previous techniques that focus on thermal phonon conduction such as reinforcing with high thermal conductivity fillers [42], controlling polymer crystallinity [26] and orienting molecular chains [27].

\section{Acknowledgements}

This work was partially supported by the Advanced Low Carbon Technology Research and Development Program of the Japan Science and Technology Agency, a Grant-in-Aid for Scientific Research (A) from Japan Society of the Promotion Science (Grant Number 16H02117), and the Tohoku University Nanofabrication Platform in Nanotechnology Platform Project sponsored by the Ministry of Education, Culture, Sports, Science and Technology. We also thank Okitsumo Inc. for their helpful discussions and financial support.

\section{References}

[1] The International Technology Roadmap for Semiconductors (ITRS), http://www.itrs.net/reports.html.

[2] R. Mahajan, C. P. Chiu, and G. Chrysler, "Cooling a microprocessor chip," Proc. IEEE, vol. 94, no. 8, pp. 1476-1485, 2006.

[3] A. L. Moore and L. Shi, "Emerging challenges and materials for thermal management of electronics," Mater. Today, vol. 17, no. 4, pp. 163-174, 2014.

[4] E. Yablonovitch, "Inhibited spontaneous emission in solid-state physics and electronics," Phys. Rev. Lett., vol. 58, no. 20, pp. 2059-2062, 1987.

[5] S. Maruyama, T. Kashiwa, H. Yugami, and M. Esashi, "Thermal radiation from two-dimensionally confined modes in microcavities," Appl. Phys. Lett., vol. 79, no. 9, pp. 1393-1395, 2001.

[6] Y. X. Yeng, M. Ghebrebrhan, P. Bermel, W. R. Chan, J. D. Joannopoulos, M. Soljačić, and I. Celanovic, "Enabling high-temperature nanophotonics for energy applications.," Proc. Natl. Acad. Sci. U. S. A., vol. 109, no. 7, pp. 2280-2285, 2012.

[7] K. A. Arpin, M. D. Losego, A. N. Cloud, H. Ning, J. Mallek, N. P. Sergeant, L. Zhu, Z. Yu, B. Kalanyan, G. N. Parsons, G. S. Girolami, J. R. Abelson, S. Fan, and P. V. Braun, "Three-dimensional self-assembled photonic crystals with high temperature stability for thermal emission modification," Nat. Commun., vol. 4, p. 2630, 2013.

[8] M. Garín, D. Hernández, T. Trifonov, and R. Alcubilla, "Three-dimensional metallo-dielectric selective thermal emitters with high-temperature stability for thermophotovoltaic applications," Sol. Energy Mater. Sol. Cells, vol. 134, pp. 22-28, 2015.

[9] L. P. Wang, B. J. Lee, X. J. Wang, and Z. M. Zhang, "Spatial and temporal coherence of thermal radiation in asymmetric Fabry-Perot resonance cavities," Int. J. Heat Mass Transf., vol. 52, no. 13-14, pp. 3024-3031, 2009.

[10] N. P. Sergeant, O. Pincon, M. Agrawal, and P. Peumans, "Design of wide-angle solar-selective absorbers using aperiodic metal-dielectric stacks.," Opt. Express, vol. 17, no. 25, pp. 22800-22812, 2009.

[11] A. V Zayats, I. I. Smolyaninov, and A. A. Maradudin, "Nano-optics of surface plasmon polaritons," Phys. Rep., vol. 408, pp. 131-314, 2005. 
[12] J.-J. Greffet, R. Carminati, K. Joulain, J.-P. Mulet, S. Mainguy, and Y. Chen, “Coherent emission of light by thermal sources," Nature, vol. 416, no. 7, pp. 61-64, 2002.

[13] H. Sai, H. Yugami, K. Nakamura, N. Nakagawa, H. Ohtsubo, and S. Maruyama, "Selective emission of Al 2 O 3/Er 3 Al 5 O 12 eutectic composite for thermophotovoltaic generation of electricity," Jpn. J. Appl. Phys., vol. 39, no. 4, pp. 1957-1961, 2000.

[14] T. Inoue, M. De Zoysa, T. Asano, and S. Noda, "Single-peak narrow-bandwidth mid-infrared thermal emitters based on quantum wells and photonic crystals,” Appl. Phys. Lett., vol. 102, no. 19, pp. 10-14, 2013.

[15] H. Sai and H. Yugami, "Thermophotovoltaic generation with selective radiators based on tungsten surface gratings," Appl. Phys. Lett., vol. 85, no. 16, pp. 3399-3401, 2004.

[16] A. Lenert, D. Bierman, Y. Nam, W. Chan, I. Celanovic, M. Soljacic, and E. Wang, “A nanophotonic solar thermophotovoltaic device," Nat. Nanotechnol., vol. 9, no. 2, pp. 126-130, 2014.

[17] S. Ogawa, K. Okada, N. Fukushima, and M. Kimata, "Wavelength selective uncooled infrared sensor by plasmonics," Appl. Phys. Lett., vol. 100, no. 2, 2012.

[18] H. T. Miyazaki, K. Ikeda, T. Kasaya, K. Yamamoto, Y. Inoue, K. Fujimura, T. Kanakugi, M. Okada, K. Hatade, and S. Kitagawa, "Thermal emission of two-color polarized infrared waves from integrated plasmon cavities," Appl. Phys. Lett., vol. 92, no. 14, pp. 2006-2009, 2008.

[19] C. G. Granqvist and A. Hjortsberg, "Radiative cooling to low temperatures : General considerations and application to selectively emitting SiO films," J. Appl. Phys., vol. 52, no. 6, pp. 4205-4220, 1981.

[20] A. P. Raman, M. A. Anoma, L. Zhu, E. Rephaeli, and S. Fan, "Passive radiative cooling below ambient air temperature under direct sunlight," Nature, vol. 515, no. 27, pp. 540-544, 2014.

[21] M. Shimizu and H. Yugami, "Thermal radiation control by surface gratings as an advanced cooling system for electronic devices,” J. Therm. Sci. Tech. Jpn., vol. 6, no. 2, pp. 297-306, 2011.

[22] S. Tsuda, M. Shimizu, F. Iguchi, and H. Yugami, "Thermal Management Technique Using Control of Thermal Radiation Spectrum for Encapsulated Electronic Devices," IEEE Trans. Compon., Packag. Manuf. Technol., Vol. 5, No. 7, pp.971-979, 2015.

[23] L. L. Vasiliev, “Heat pipes in modern heat exchangers,” Appl. Therm. Eng., vol. 25, no. 1, pp. 1-19, 2005.

[24] R. Singh, A. Akbarzadeh, and M. Mochizuki, "Thermal Potential of Flat Evaporator Miniature Loop Heat Pipes for Notebook Cooling," IEEE Trans. Compon. Packag. Technol., vol. 33, no. 1, pp. 32-45, 2010.

[25] M. Smalc, G. Chen, G. Shives, J. Norley, and R. A. Reynolds III, “Thermal Performance of Natural Graphite Heat Spreaders," Proceedings of IPACK, Interpack 2005-73073, pp. 1-11, 2005.

[26] Z. Han and A. Fina, "Thermal Conductivity of Carbon Nanotubes and their Polymer Nanocomposites : A Review.” Prog. Polym. Sci., vol. 36, no. 7, pp. 1-113, 2010.

[27] V. Singh, T. L. Bougher, A. Weathers, Y. Cai, K. Bi, M. T. Pettes, S. A. McMenamin, W. Lv, D. P. Resler, T. R. Gattuso, D. H. Altman, K. H. Sandhage, L. Shi, A. Henry, and B. A. Cola, "High thermal conductivity of chain-oriented amorphous polythiophene," Nat. Nanotechnol., vol. 9, no. 5, pp. 384-390, 2014.

[28] A. Henry, “Thermal transport in polymer,” Ann. Rev. Heat Transfer, pp. 485-520, 2014.

[29] F. Jiang, J. L. de Ris, and M. M. Khan, "Absorption of thermal energy in PMMA by in-depth radiation," Fire Saf. J., vol. 44, no. 1, pp. 106-112, 2009.

[30] S. Lang, M. Tschikin, S. A. Biehs, A. Y. Petrov, and M. Eich, "Large penetration depth of near-field heat flux in hyperbolic media," Appl. Phys. Lett., vol. 104, no. 12, 2014.

[31] G. Linteris, M. Zammarano, B. Wilthan, and L. Hanssen, "Absorption and reflection of infrared radiation by polymers in fire-like environments," Fire Mater., vol. 36, no. 7, pp. 537-553, 2012.

[32] A. Fina, J. Feng, and F. Cuttica, "In-depth radiative heat transmittance through polypropylene / nanoclay composites,” Polym. Degrad. Stab., vol. 98, no. 5, pp. 1030-1035, 2013.

[33] J. R. Howell, M. P. Menquc, and R Siegel, “Thermal Radiaiton Heat Transfer, 6th edition”, CRC Press, 2015.

[34] S. Basu and M. Francoeur, "Penetration depth in near-field radiative heat transfer between metamaterials," Appl. Phys. Lett., vol. 99, no. 14, pp. 2011-2014, 2011.

[35] L. Dombrovsky, J. Randrianalisoa, D. Baillis, and L. Pilon, "Use of Mie theory to analyze experimental data to identify infrared properties of fused quartz containing bubbles,” Appl. Opt., vol. 44, no. 33, pp. 7021-7031, 2005 . 
[36] L. Wang, J. I. Eldridge, and S. M. Guo, "Comparison of different models for the determination of the absorption and scattering coefficients of thermal barrier coatings," Acta Mater., vol. 64, pp. 402-410, 2014.

[37] M. Dubois, "Absolute optical absorption spectra in graphite epoxy by Fourier transform infrared photoacoustic spectroscopy,” Opt. Eng., vol. 32, no. 9, pp. 2255-2260, 1993.

[38] J. H. Kennedy and K. W. Frese, "Photooxidation of Water at Alpha-Fe2O3 Electrodes," J. Electrochem. Soc., vol. 125, no. 5, pp. 709-714, 1978.

[39] Y.-S. Chen, K.-H. Chien, C.-C. Wang, T.-C. Hung, Y.-M. Ferng, and B.-S. Pei, "Investigations of the Thermal Spreading Effects of Rectangular Conduction Plates and Vapor Chamber,” J. Electron. Packag., vol. 129, no. 3, p. 348, 2007.

[40] R.S. Bretzlaff and R. P. Wool, "Frequency Shifting and Asymmetry in Infrared Bands of Stressed Polymers," Macromolecules, vol. 16, no. 12, pp. 1907-1917, 1983.

[41] L. Zhu, A. Raman, K. X. Wang, M. A. Anoma, and S. Fan, "Radiative cooling of solar cells," Optica, vol. 1, no. 1, pp. 32-38, 2014.

[42] X. Huang, P. Jiang, and T. Tanaka, "A review of dielectric polymer composites with high thermal conductivity,” IEEE Electr. Insul. Mag., vol. 27, no. 4, pp. 8-16, 2011. 


\section{Artwork with captions}

(a)

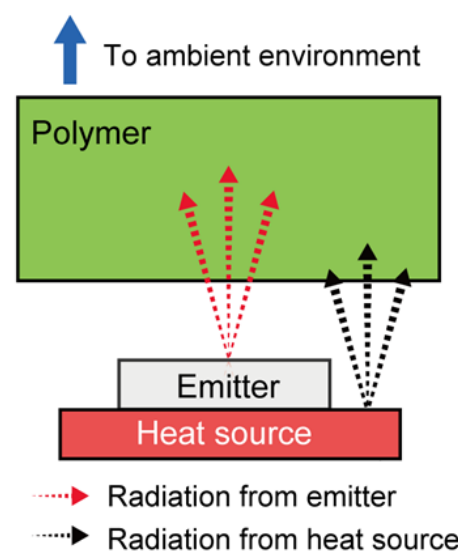

(b)

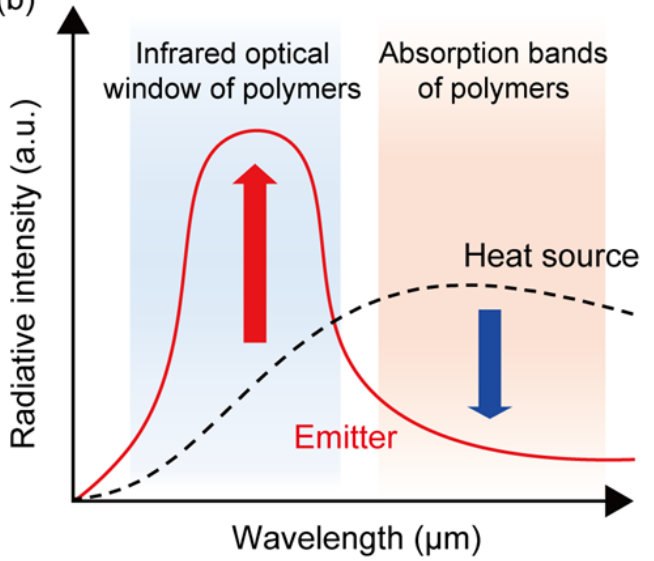

Fig. 1. (a) Schematic showing how spectral control of thermal radiation enhances heat transport in a polymer. (b) Thermal radiation spectrum from the spectrally selective emitter and heat sources as a spectrally non-selective emitter. The thermal radiation from the spectrally selective emitter is concentrated in the infrared optical window of the polymer but it is suppressed in the polymer's absorption bands.

(a)

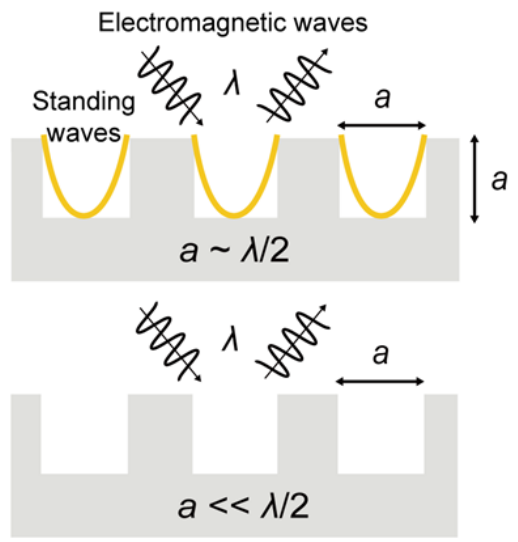

(b)

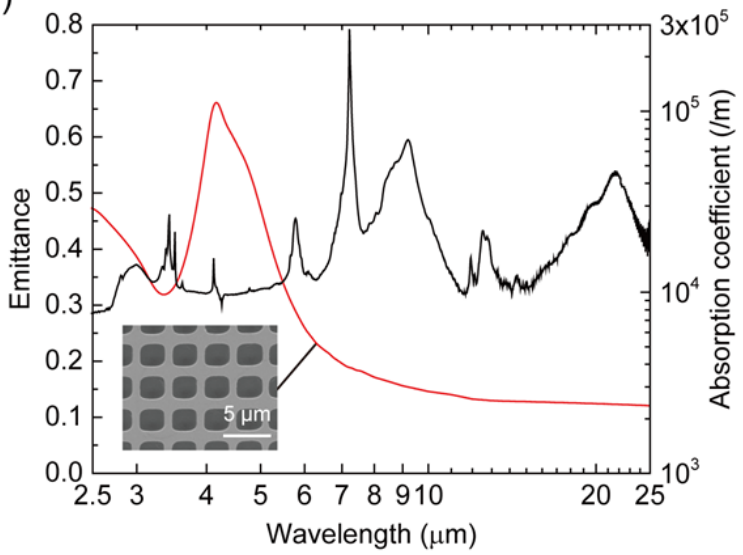

Fig. 2 (a) Schematic showing the control of thermal radiation with 2D metallic surface grating structures. In this method, thermal radiation is enhanced over a specific wavelength range by confining and amplifying electromagnetic waves with wavelengths comparable to the structure's characteristic feature size. Meanwhile, thermal radiation with wavelengths larger than the feature size is suppressed because of the inherently high reflection properties of the metallic surfaces and the diffraction limit. (b) Optical properties of the fabricated spectrally selective emitter and acrylic resin. The red line is the emittance of the spectrally selective emitter, measured by FT-IR. The black line is the absorption coefficient of the acrylic resin, estimated from the FT-IR measurement by using the $\mathrm{KBr}$ pellet technique. The insert shows the surface morphology of the spectrally selective emitter, observed with scanning electron microscopy. 


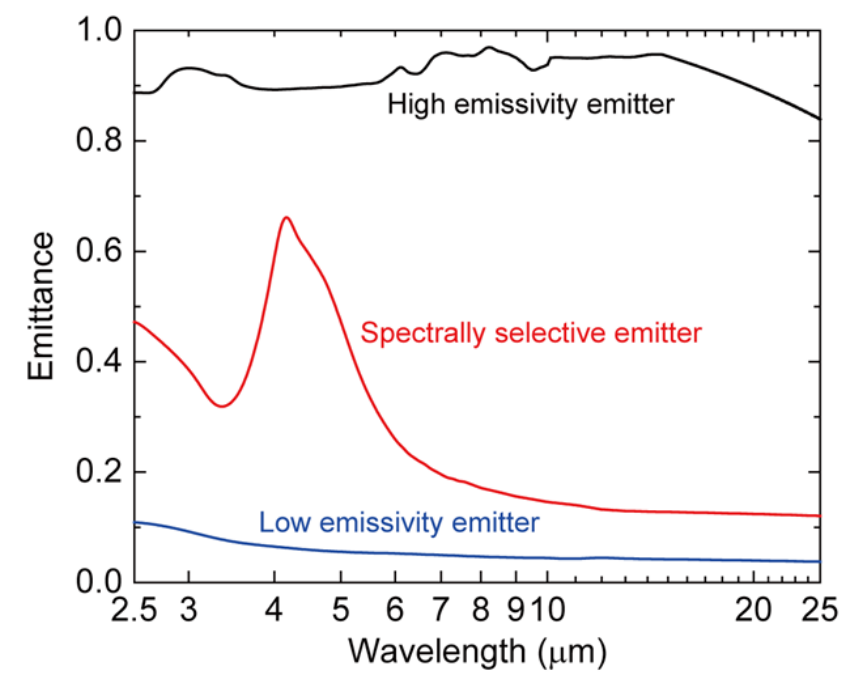

Fig. 3 The optical properties of various emitters, measured by FT-IR.
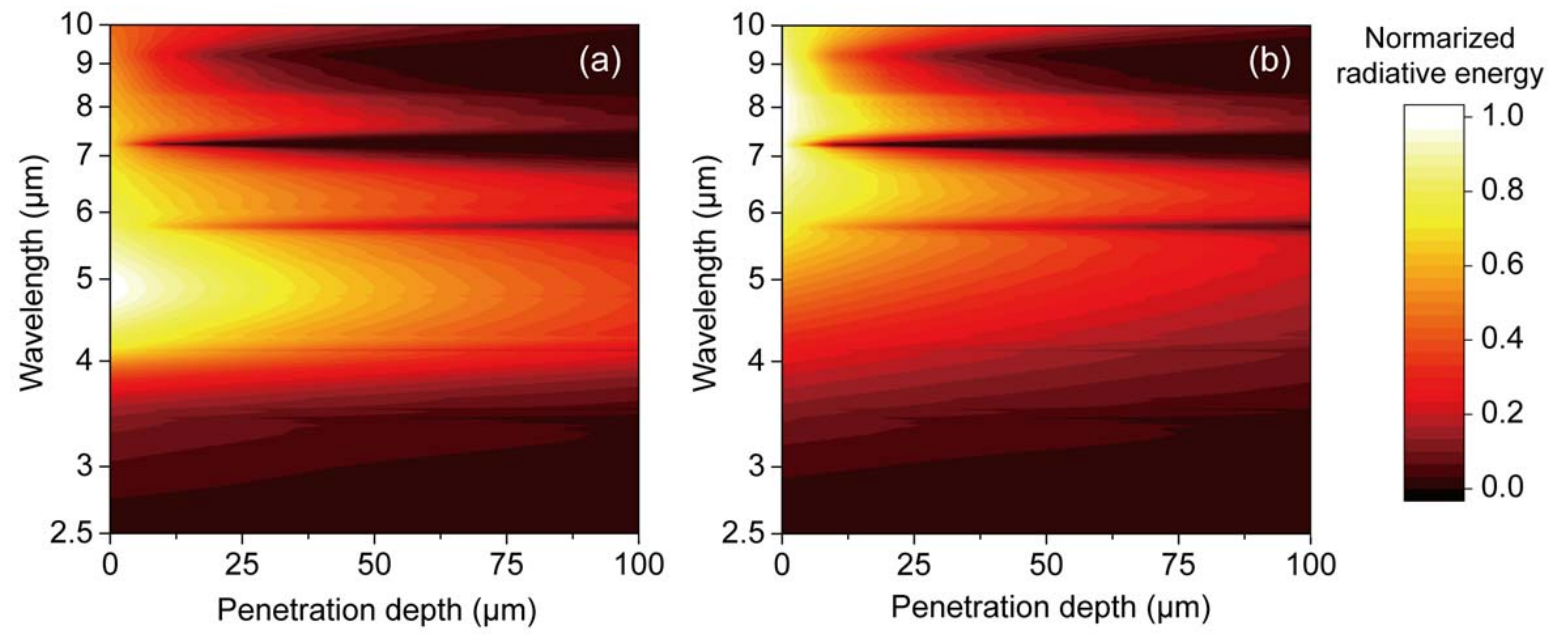

Fig. 4 Penetration depth of thermal radiation into polymers as a function of wavelength for two emitters: (a) spectrally selective emitter, (b) high emissivity emitter. The radiative energy with each emitter is normalized by the maximum value. The radiation propagation medium is assumed to be acrylic resin. The optical properties to calculate the penetration depth of thermal radiation correspond to the measured values. 


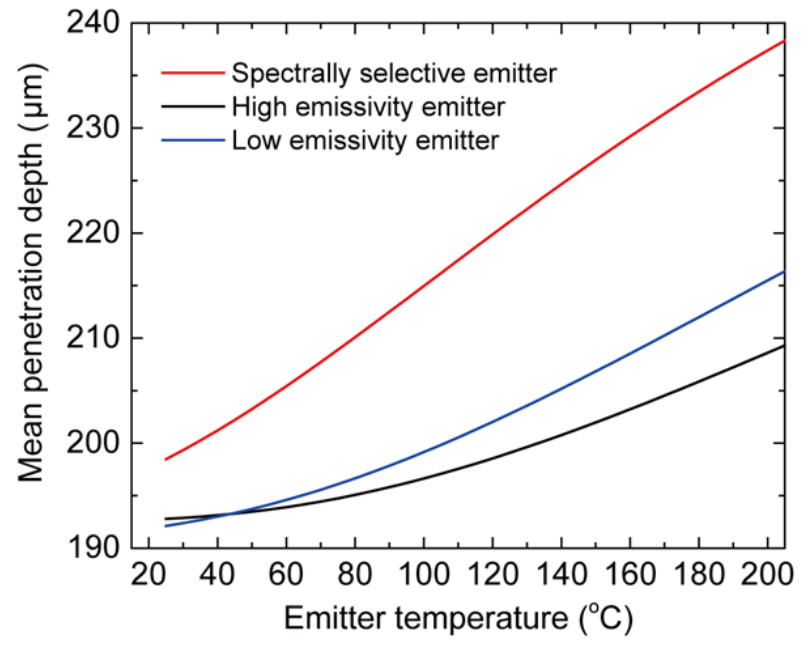

Fig. 5 Mean penetration depth of thermal radiation, as a function of emitter temperature, for various emitters. The radiation propagation medium is assumed to be acrylic resin. The optical properties used to calculate the mean penetration depth of thermal radiation correspond to the measured values.

(a)

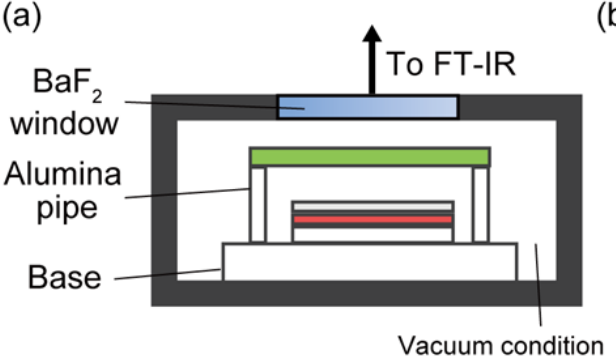

(b)

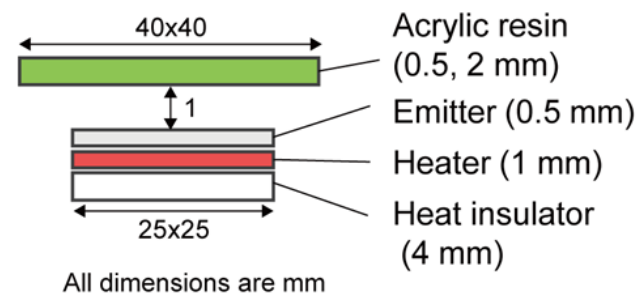

(c)

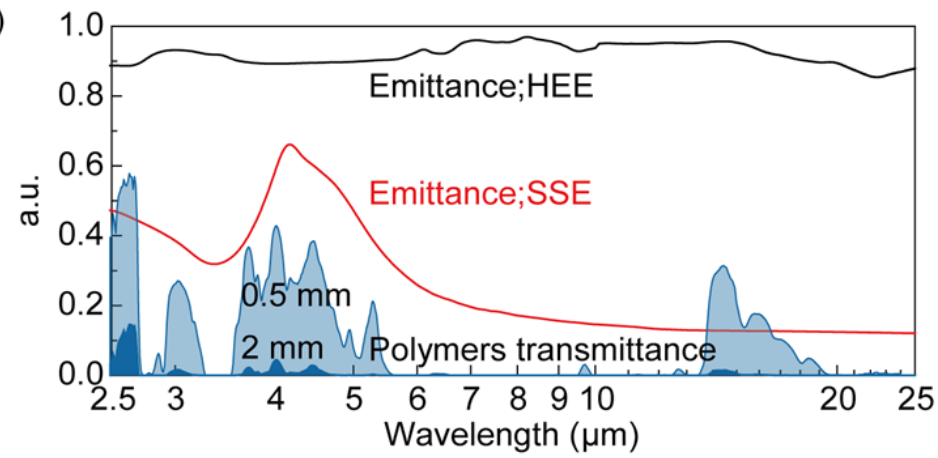

Fig. 6 Experimental configuration: (a) experimental equipment in a vacuum chamber. The radiative energy through the polymer plate from the emitter is measured by an optical system with FT-IR, and the surface temperature distribution of the polymer plate is evaluated by a thermal camera. The infrared window has a diameter of $30 \mathrm{~mm}$; (b) the details of the components near the heat source; (c) the optical properties of the spectrally selective emitter and high emissivity emitter. The red and black lines denote the emittance of each emitter. The blue and deep blue regions are the external transmittance of acrylic resin with a thickness of $0.5 \mathrm{~mm}$ and $2 \mathrm{~mm}$, respectively. 

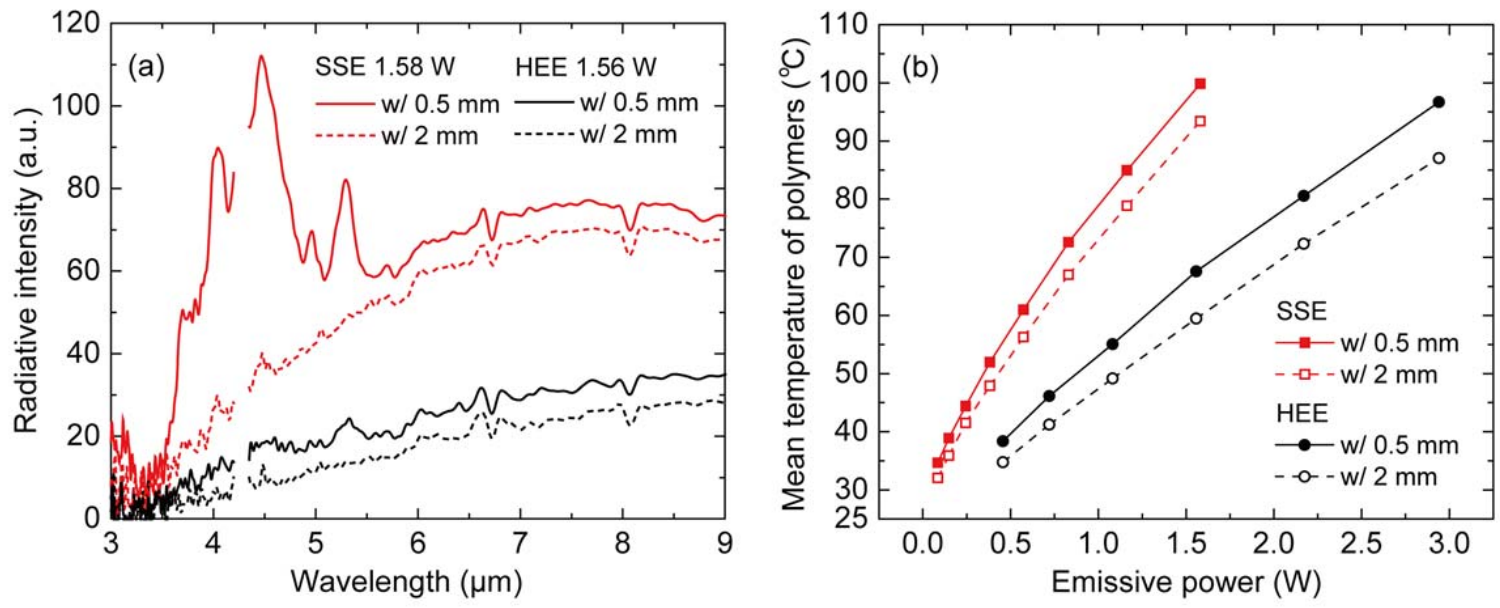

Fig. 7 (a) Radiative spectra through polymer plates from two emitters. (b) Mean surface temperatures of polymer plates as a function of emissive power from two emitters. The SSE and HEE are the spectrally selective emitter and high emissivity emitter, respectively.
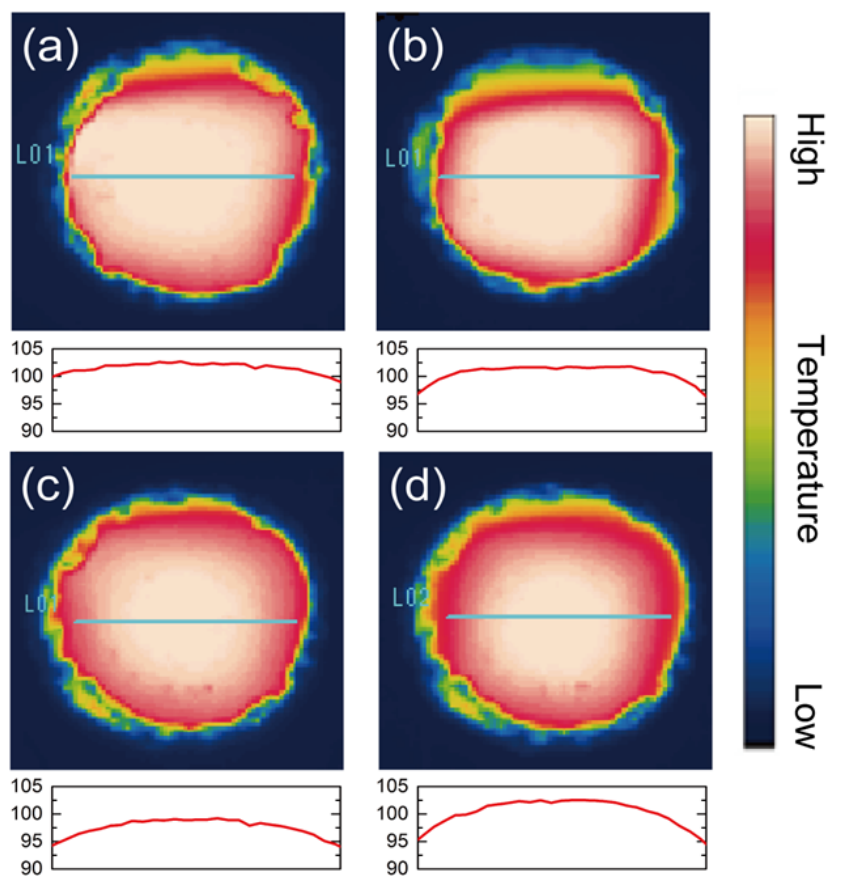

Fig. 8 Thermal images of the surfaces of polymer plates through the infrared optical window: (a) spectrally selective emitter with polymer plate (thickness of $0.5 \mathrm{~mm}$ ); (b) high emissivity emitter with polymer plate (thickness of $0.5 \mathrm{~mm}$ ); (c) spectrally selective emitter with polymer plate (thickness of $2 \mathrm{~mm}$ ); (d) high emissivity emitter with polymer plate (thickness of $2 \mathrm{~mm}$ ). The line profiles under the thermal images show the temperature profiles of the blue lines in the thermal images. The hot regions include the area of the emitters as compared with the area of the components: the window diameter is $30 \mathrm{~mm}$, and the emitter size is a $20-\mathrm{mm}$ square. 


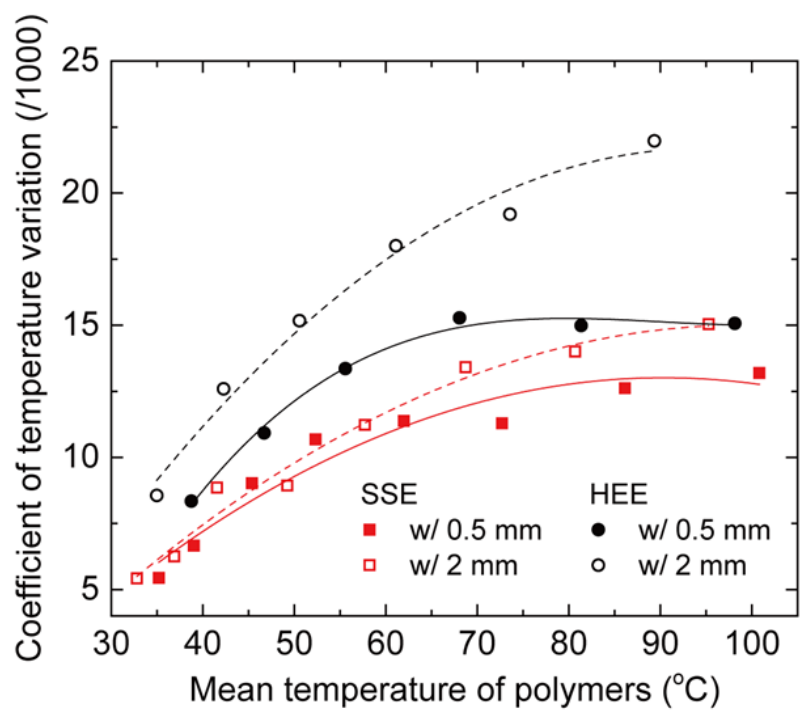

Fig. 9 Coefficient of temperature variation for each emitter and polymer plates with two thicknesses, $0.5 \mathrm{~mm}$ and $2 \mathrm{~mm}$, as a function of the mean surface temperature of the polymer plates. The SSE and HEE are the spectrally selective emitter and high emissivity emitter, respectively. The solid and hollow symbols denote the thicknesses of the acrylic resin: $0.5 \mathrm{~mm}$ and $2 \mathrm{~mm}$, respectively. The lines are guides for the eye.

\section{Highlights}

- A new way to improve heat transfer in polymers by thermal radiation is proposed.

- A selective thermal emitter is produced, made of a metallic diffraction grating.

- Controlling the thermal radiation boosted its propagation deep into polymers.

- Effectively propagating thermal radiation in polymers improved their heat spreading. 